\title{
Correction to: Prevalence of pica and rumination behaviors in German children aged 7-14 and their associations with feeding, eating, and general psychopathology: a population-based study
}

\author{
Andrea S. Hartmann ${ }^{1}$ (D) Tanja Poulain ${ }^{2,3} \cdot$ Mandy Vogel $^{2,3} \cdot$ Andreas Hiemisch $^{2,3} \cdot$ Wieland Kiess $^{2,3} \cdot$ Anja Hilbert $^{4}$
}

Published online: 8 June 2018

○) Springer-Verlag GmbH Germany, part of Springer Nature 2018

\section{Correction to: European Child \& Adolescent Psychiatry https://doi.org/10.1007/s00787-018-1153-9}

In the original publication, under the discussion section, the prevalence of recurrent rumination behavior is stated as $2.94 \%$ as opposed to the correct $1.49 \%$ described in the abstract and the results.

The original article can be found online at https://doi.org/10.1007/ s00787-018-1153-9.

Andrea S. Hartmann

andrea.hartmann@uos.de

1 Institute of Psychology, Department of Human Sciences, Osnabrück University, Knollstrasse 15, 49069 Osnabrück, Germany

2 LIFE Leipzig Research Center for Civilization Diseases, University of Leipzig, Philipp-Rosenthal-Strasse 27, 04103 Leipzig, Germany

3 Center for Pediatric Research, Hospital for Children and Adolescents, University of Leipzig Medical Center, Liebigstrasse 20a, 04103 Leipzig, Germany

4 Department of Medical Psychology and Medical Sociology, Department of Psychosomatic Medicine and Psychotherapy, Integrated Research and Treatment Center Adiposity Diseases, University of Leipzig Medical Center, Philipp-Rosenthal-Strasse 27, 04103 Leipzig, Germany 